\title{
Multiple gene knock-down by a single lentiviral vector expressing an array of short hairpin RNAs
}

\author{
Veronique Stove \\ Department of Clinical Chemistry \\ Microbiology and Immunology \\ Ghent University \\ Ghent University Hospital \\ 4BlokA, De Pintelaan 185 \\ 9000 Ghent, Belgium \\ Tel: 3292405871 \\ Fax: 3292404985 \\ E-mail: Veronique.Stove@UGent.be \\ Kaatje Smits \\ Department of Clinical Chemistry \\ Microbiology and Immunology \\ Ghent University \\ Ghent University Hospital \\ 4BlokA, De Pintelaan 185 \\ 9000 Ghent, Belgium \\ Tel: 3292405310 \\ Fax: 3292403659 \\ E-mail: Kaatje.Smits@UGent.be
}

\section{Evelien Naessens}

Department of Clinical Chemistry Microbiology and Immunology Ghent University Ghent University Hospital 4BlokA, De Pintelaan 185 9000 Ghent, Belgium

Tel: 3292405310

Fax: 3292403659

E-mail: Evelien.Naessens@UGent.be

\author{
Jean Plum \\ Department of Clinical Chemistry \\ Microbiology and Immunology \\ Ghent University \\ Ghent University Hospital \\ 4BlokA, De Pintelaan 185 \\ 9000 Ghent, Belgium \\ Tel: 3292403641 \\ Fax: 3292403659 \\ E-mail: Jean.Plum@UGent.be

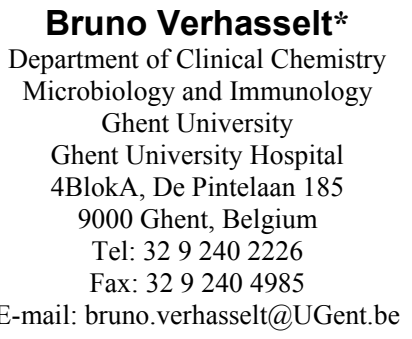

Financial support: This work was supported by grants from the Research Foundation - Flanders (FWO). V.S. and K.S. equally contributed and are Ph.D. fellows of the FWO, and B.V. is a Senior Clinical Investigator of the FWO.

\footnotetext{
* Corresponding author
} 
Abbreviations: B2M: $\beta 2$-microglobulin

CLTC: clathrin heavy chain

EGFP: enhanced green fluorescent protein

GTPases: guanosine triphosphatases

pol III: polymerase III

RNAi: RNA interference

SDF-1 $\alpha$ : stromal cell-derived factor-1 alpha

shRNA: short hairpin RNA

siRNAs: small interfering RNAs
RNA interference (RNAi), mediated by short doublestranded RNAs, is a powerful mechanism for posttranscriptional gene silencing. Sustained expression of short hairpin RNA (shRNA) can be accomplished in mammalian cells by viral delivery systems. Using lentiviral constructs, stable gene silencing is established both in dividing and non-dividing cells. Targeting one single gene can lead to the development of escape mutants or may be insufficient to silence redundant pathways. Therefore, simultaneous targeting of multiple genes may be necessary. We have generated a lentiviral vector-based system for expression of multiple shRNAs from a single viral vector, which also encodes an EGFP reporter protein. We show that knock-down of each single gene from multiple target vectors is achieved at an efficiency comparable to that obtained after transduction using single target viral vectors. In this way, we were able to knock-down several members of the human Rho-family GTPases in T cells. Double and triple knock-down persisted after multiple passages of the cells. The ability to inhibit two or more genes simultaneously from one single expression vector further widens the application spectrum of RNAi, both in functional studies and therapeutic strategies.

Knock-down by small interfering RNAs (siRNAs) has been shown to be a powerful technique. Transfer of siRNA to cells can be achieved in several ways, e.g. delivered exogenously or expressed endogenously from RNA polymerase III (pol III) promoters (reviewed by Dykxhoorn et al. 2003). Initial reports using siRNA in mammalian cells focused on the transient knock-down of target gene expression, using direct transfection of synthetic siRNA, or transient expression of double stranded RNA via transfection of plasmid DNA, bearing a RNA pol III promoter-driven expression cassette.

Several reports have also shown the feasibility to knock down several proteins simultaneously (Anderson et al. 2003; Yu et al. 2003; Fitzgerald et al. 2004; Lakka et al. 2004). Moreover, the combination of siRNAs directed against different regions of a single gene can be used to improve the gene silencing effect or to avoid viral escape mutants (Ji et al. 2003; Gonzalez et al. 2005; Schubert et al. 2005). Multiple RNAi can be accomplished by transfection of multiple siRNA species (Ji et al. 2003; Yu et al. 2003). Alternatively, a construct with several consecutive short hairpin RNAs (shRNAs) expression cassettes (Gonzalez et al. 2005; Jazag et al. 2005; Schubert et al. 2005), or expression of a RNA that is cleaved into several shRNAs, are an option (Anderson et al. 2003; Lakka et al. 2004). For expression in primary cells or cell lines difficult to transfect, only viral transfer reaches a significant efficiency (Verma and Somia, 1997). More specifically, lentiviral vectors have been shown to be a valuable approach for stable and effective transfer of functional shRNAs in these cells (Qin et al. 2003; Rubinson et al. 2003). To extend the application possibilities of lentiviral vector mediated knock-down, we developed lentiviral vectors encoding within one construct distinct shRNAs, each driven by an individual pol III promoter. This ensures simultaneous expression of all distinct shRNAs. We show that these multiple shRNAs vectors down-modulate the respective targets at a comparable level as the single target vectors. As proof of principle we used these vectors to silence the expression of up to three Rho guanosine triphosphatases (GTPases) simultaneously. Rho GTPases are a family of regulatory proteins, shown to play a role in different functions, such as cellular motility, adhesion and spatial organization of membrane receptors and signaling molecules (Cantrell, 2003). In hematopoietic cells like T cells, several Rho GTPases, e.g. Cdc42, Rac1 and Rac2 are expressed. These proteins are highly homologous and can have overlapping activity (Cantrell, 2003). Therefore, to study their function by knock-down, it might be necessary to target several of these small GTPases simultaneously.

In this study, we have examined the functionality of different shRNA cassettes, inserted one after the other in a single lentiviral construct. We found that these recombinant lentiviruses were able to stably knock down the expression of the targeted genes on mRNA level as well as on protein level.

\section{MATERIALS AND METHODS}

\section{Constructs}

The pSUPER plasmid containing H1 RNA pol III promoter, described previously (Brummelkamp et al. 2002), was obtained from Oligoengine (Seattle, WA). A unique MunI site was inserted downstream the HindIII restriction site, creating the pSUPER(MunI) vector. The RNAi probes were $B g l I I-H i n d I I I$ cloned into this vector. To construct the 19 nucleotide hairpin siRNA cassettes, two complementary DNA oligonucleotides were chemically 


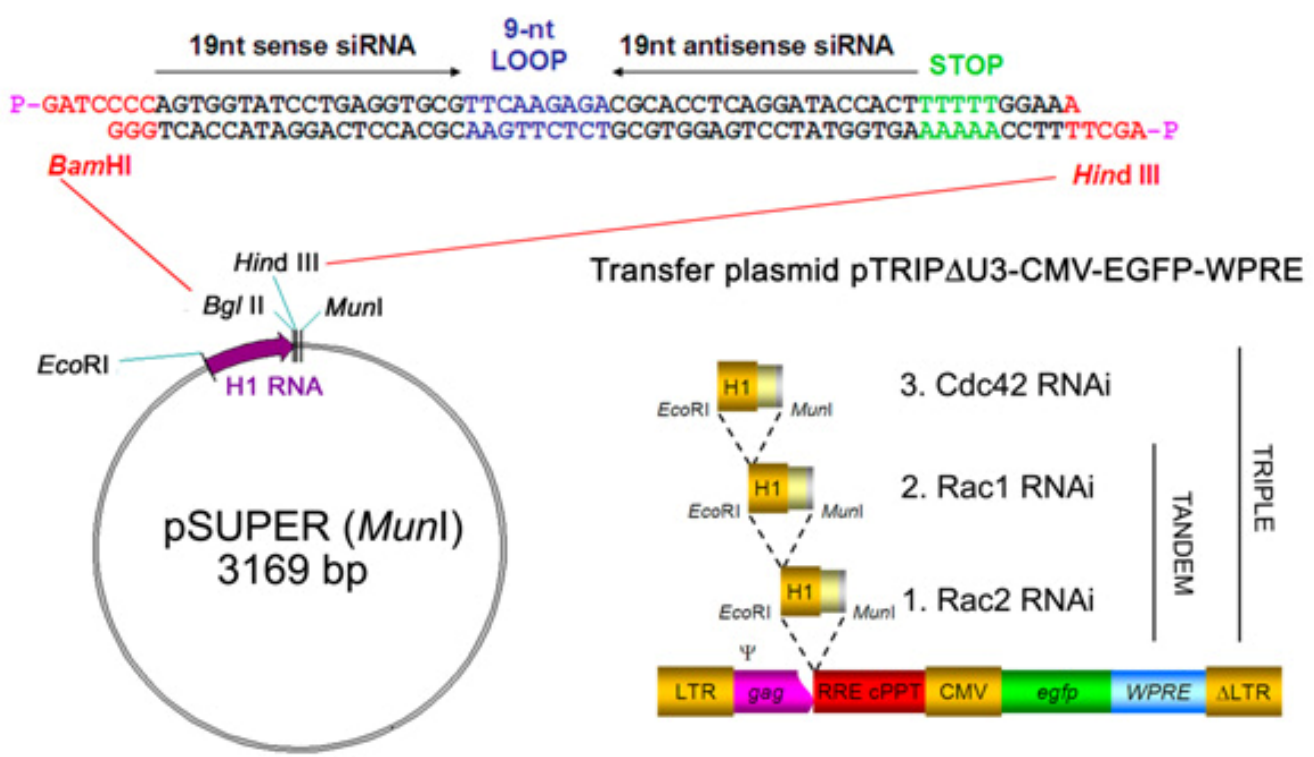

Envelope plasmid pMD.G

CMV VSV-G poly(A)
Packaging plasmid p8.91

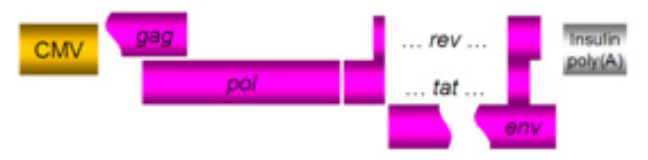

Figure 1. Representation of RNAi subcloning strategy. Modified pSUPER (Munl) vector carrying the H1-RNAi cassette: a H1-RNA promoter driving a shRNA after Bg/ll-Hindlll cloning of the encoding dsDNA oligo. From this EcoRI-Munl digested vector, the H1-RNAi cassette is cloned into EcoRI digested pTRIPAU3-CMV-EGFP-WPRE transfer plasmid, leaving one EcoRI site upstream for cloning of additional H1-RNAi cassettes. Figure shows relevant part of transfer, packaging and envelope plasmids, which are co-transfected for virus production. CMV: Cytomegalovirus minimal promoter; LTR: long terminal repeat; DLTR: partial deleted LTR; RRE: Rev responsive element; CPPT: central polypurine tract; WPRE: Woodchuck Hepatitis Virus Posttranscriptional Regulatory Element; VSV-G: Vesicular Stomatitis Virus Glycoprotein; polyA: polyadenylation signal; bp: base pairs.

synthesized (Invitrogen, Merelbeke, Belgium), annealed, and inserted immediately downstream of the $\mathrm{H} 1$ promoter: 5'-GATCCCC-19-TTCAAGAGA-19-TTTTTGGAAA-3' and 5'-AGCTTTTCCAAAAA-19-TCTCTTGAA-19-GGG3'. All siRNA sequences were BLAST searched in the National Center for Biotechnology Information's (NCBI, http://www.ncbi.nlm.nih.gov/BLAST/) "search for short nearly exact matches" mode against all human sequences deposited in the GenBank and RefSeq databases and were not found to have significant homology ( $>17$ contiguous nucleotides of identity) to genes other than the targets. The target sequences for each of the genes were as follows: Rac1 (GenBank Accession no. AF498964), 5'AGTGGTATCCTGAGGTGCG-3', corresponding to $\mathrm{nt}$ positions 287-305, Rac2 (GenBank accession no. AF498965), 5'-GGACACCATCGAGAAACTG-3', corresponding to nt positions 369-387, Cdc42 (GenBank accession no. NM_001791), 5'GCCTATCACTCCAGAGACT-3', corresponding to $\mathrm{nt}$ positions 509-527, CLTC (GenBank Accession NM_004859), 5'-GTAATCCAATTCGAAGACC-3', corresponding to nt positions 413-431 (Motley et al. 2003), was used as an irrelevant control.
Lentiviral production was done as described before (Stove et al. 2005) using packaging plasmid p8.91 (Zufferey et al. 1997), vesicular stomatitis virus (VSV) envelopeexpression plasmid (pMD.G) (Naldini et al. 1996) and TRIPAU3-CMV-EGFP-WPRE transfer vector. As MunI and EcoRI generate compatible sticky ends, the H1-RNAi cassettes could be EcoRI-MunI transferred from the pSUPER(MunI) vector into the EcoRI digested TRIP $\Delta$ U3CMV-EGFP-WPRE vector. The EcoRI remains a unique restriction site after ligation and can still be used to subclone additional H1-RNAi cassettes into the lentiviral vector (Figure 1).

\section{Cell culture and viral production}

All the cells (SupT1 (AIDS Research and Reference Reagent Program, NIH, Bethesda, MD) and 293T (American Type Culture Collection, Rockville, MD)) were grown in Iscove's modified Dulbecco's medium (IMDM, Invitrogen) supplemented with penicillin (100 IU/ml, Invitrogen), streptomycin $(100 \mu \mathrm{g} / \mathrm{ml}$, Invitrogen $)$ and $10 \%$ heat-inactivated fetal calf serum (PerBio,Erembodegem, Belgium). VSV-glycoprotein pseudotyped lentiviral vector 
particles were produced by co-transfection of 293T cells with transfer, packaging and envelope plasmids (respectively $20 \mu \mathrm{g}, 20 \mu \mathrm{g}$ and $10 \mu \mathrm{g} / 175 \mathrm{~cm}^{2}$ culture flask) using a Calcium Phosphate Transfection Kit (Invitrogen) and viral sup was harvested $40 \mathrm{hrs}$ later. Viral supernatants contained at least $10^{8}$ transducing units per $\mathrm{ml}$ titrated on SupT1 cells. Transductions were carried out in the presence of polybrene $(4 \mu \mathrm{g} / \mathrm{ml}$; Sigma-Aldrich, Bornem, Belgium) with multiplicity of infection (MOI) well above 1.

\section{Chemotaxis}

Chemotaxis assays were performed in duplicate using $5 \mu \mathrm{m}$ pore filters (Transwell, 24 well cell cluster, Corning Costar, Cambridge, MA) within 10 days after transduction. In order to have an internal negative control for migration in each well, transduced cells were spiked with non-transduced cells to a final ratio of $50 \% \mathrm{EGFP}^{+} / 50 \% \mathrm{EGFP}^{-}$cells. Migration medium $(600 \mu \mathrm{l}$ IMDM supplemented with penicillin $(100 \mathrm{IU} / \mathrm{ml})$, streptomycin $(100 \mu \mathrm{g} / \mathrm{ml})$ and $0.5 \%$ bovine serum albumin (Sigma)) containing $10 \mathrm{ng} / \mathrm{ml}$ recombinant human (SDF-1 $\alpha /$ CXCL12; Peprotech, London, UK) was added to the lower compartment. Cell suspension $\left(5.10^{6}\right.$ cells $/ \mathrm{ml}$ in $\left.100 \mu \mathrm{l}\right)$ was placed in the upper well. Transwells were incubated for $3 \mathrm{hrs}$ at $37^{\circ} \mathrm{C}, 7 \%(\mathrm{v} / \mathrm{v}) \mathrm{CO}_{2}$.

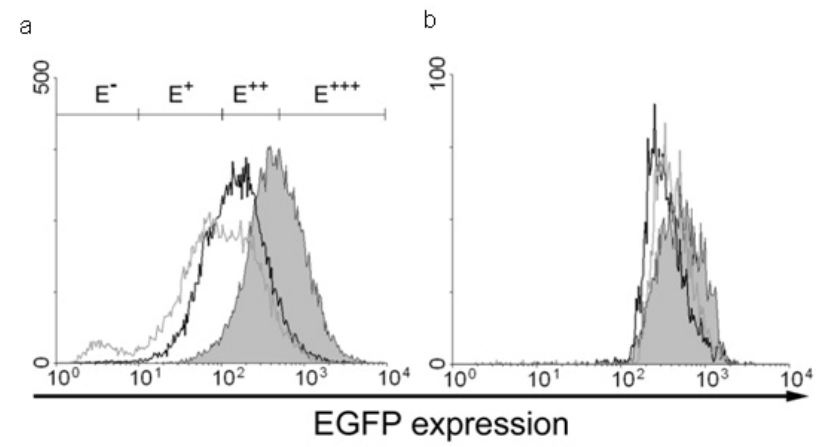

Figure 2. EGFP expression in transduced SupT1 cells. Flow cytometric EGFP expression profile of SupT1 cells, transduced with virus encoding single Rac2 shRNA (filled histograms), tandem Rac2 and Rac1 shRNA (black line) and triple Cdc42, Rac2 and Rac1 shRNA (grey line) before (a) and after (b) sorting for EGFP expression. Markers $\mathrm{E}^{-}$to $\mathrm{E}^{+++}$indicate expression intervals used in chemotaxis experiments (see text and Figure $5)$.

Upper wells were removed and cells migrated into the lower compartment were harvested after addition of a fixed amount of Flow-Count Fluorospheres (Beckman Coulter, Fullerton, CA). Flow cytometry was done on a FACS ${ }^{\circledR}$ Calibur (Becton Dickinson Immunocytometry Systems, Mountain View, CA) to determine the absolute number of input and migrated cells as well as the relative frequency of $\mathrm{EGFP}^{+}$cells in initial and migrated population. Percentage of migrating cells was calculated as follows: $\left(\% \mathrm{EGFP}^{+}\right.$cells in migrated population $\mathrm{x}$ total amount of migrated cells) / $\left(\%\right.$ EGFP $^{+}$cells in initial population $\mathrm{x}$ total amount of input cells).

\section{Real-time PCR}

Total RNA was extracted from sorted shRNA transduced cells as instructed by supplier (TRIzol, Invitrogen), DNasetreated (DNase I, Invitrogen) and reverse transcribed (Reverse Transcription Core Kit, Eurogentec, Seraing, Belgium). Genes assayed included Rac1, Rac2 and Cdc42 (TaqMan detection chemistry, Eurogentec) and $\beta 2$ microglobulin housekeeping gene (B2M) (Sybr Green I detection, Eurogentec). Primers (Invitrogen) and Taqman probes (Eurogentec) were described previously (Kovacic et al. 2001; Vandesompele et al. 2002) or were designed with Primer Express 2.0 software (Applied Biosystems, Foster City, CA). Sequences were as follows (5' to $\left.3^{\prime}\right)$ : Rac1 (Kovacic et al. 2001) forward CCCTATCCTATCCGCAAACA; reverse CGCACCTCAGGATACCACTT; probe FAMTCCCTTGTGAGTCCTGCATCA-TAMRA; Rac2 forward TCCGGCCGCTCTCCTATC; reverse CGCACTTCTGGGAACCACTT; probe FAMTCCTCATCTGCTTCTCCTCGTCAGC-TAMRA; Cdc42 forward CGTGACCTGAAGGCTGTCAA; reverse ACACACCTGCGGCTCTTCTT; probe FAMTTGCTTCGTCAAATACATTCTTTAGGCCTTTCTG-

TAMRA; B2M (Vandesompele et al. 2002) forward TGCTGTCTCCATGTTTGATGTATCT; reverse TCTCTGCTCCCCACCTCTAAGT. Dyes used were 6carboxyfluorescein (FAM) and 6-carboxytetramethylrhodamine (TAMRA). Quantitative real-time PCR was performed on ABI Prism 7000 Sequence Detection System (Applied Biosystems). Cycle conditions were $10 \mathrm{~min}$ at $95^{\circ} \mathrm{C}, 40$ cycles of $95^{\circ} \mathrm{C}$ for $15 \mathrm{sec}$ and $60^{\circ} \mathrm{C}$ for 1 min. For each PCR run with Sybr Green I, a melting curve analysis was performed to guarantee the amplification specificity. All amplifications were done in triplicate runs and included a standard curve of serial dilutions of total thymus cDNA, no-template control and samples of approximately 25 ngeq RNA, all in triplicate. For genes assayed with TaqMan detection chemistry, reaction mixture contains $900 \mathrm{nM}$ primer and $200 \mathrm{nM}$ probe. For genes assayed with Sybr Green I, final primer concentration is $300 \mathrm{nM}$.

\section{Western blotting}

Sorted transduced cells were lysed in Laemmli sample buffer (0.125 M Tris [pH 6.8], 25\% Glycerol, 2.3\% SDS), and the protein concentrations were determined by $R C D C$ Protein Assay (Bio-Rad, Nazareth, Belgium). After adding 5\% 2-mercapto-ethanol and boiling, equal amounts of protein were run on a $12 \%$ precast Novex Tris-Glycine polyacrylamide gel (Invitrogen) in reducing conditions and blotted onto nitrocellulose membranes (Amersham Biosciences, Roosendaal, The Netherlands). Quenching and immunostaining of the blots was done in 5\% non-fat dry milk in phosphate-buffered saline containing $0.5 \%$ Tween20. The membranes were quenched for $1 \mathrm{hr}$, incubated with primary antibody $(\mathrm{Ab})$ for $1 \mathrm{hr}$, washed four times for 10 min, incubated with horseradish peroxidase-conjugated secondary $\mathrm{Ab}$ (Amersham Biosciences) for $45 \mathrm{~min}$ and washed six times for 10 min. Primary Abs used included 
anti-Rac $\mathrm{Ab}$ (mouse, monoclonal, Upstate Biotechnology, Waltham, MA), anti-Rac2 Ab (rabbit polyclonal, C-11, Santa Cruz Biotechnology, Santa Cruz, CA) and anti$\mathrm{Cdc} 42 \mathrm{Ab}$ (P1, Santa Cruz). Detection was done using enhanced chemiluminescence reagent (Amersham Biosciences) or Supersignal West Femto (Pierce, Rockford, IL) as a substrate. For normalization of lysate loading, immunostaining with a mouse anti-Actin antibody (mouse monoclonal, cloneC4, ICN, Aurora, $\mathrm{OH}$ ) was performed (not shown). Quantification was done using an Image Station 440CF chemoluminescence imager (Kodak, Hemel Hempstead, UK) and Quantity One software (Bio-Rad).

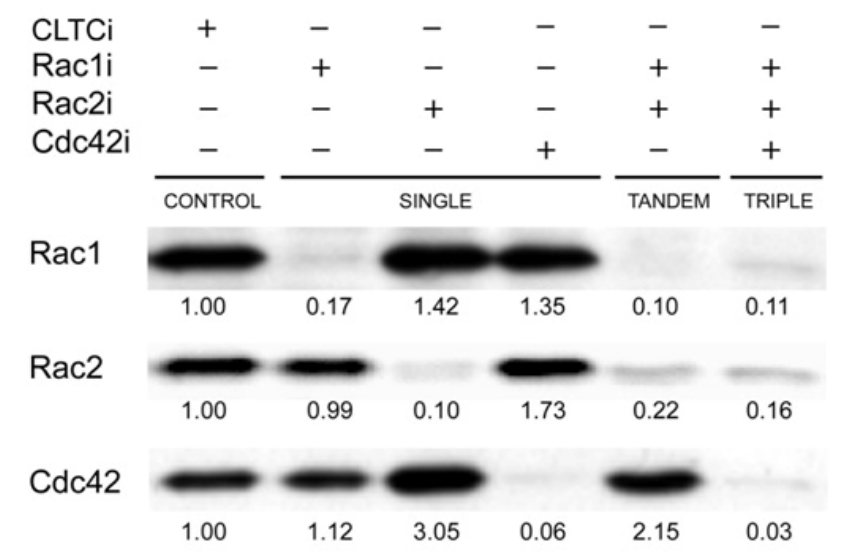

Figure 3. Immunoblot of lysates from sorted SupT1 cells, expressing specific RNAi as indicated. Each row represents blot stained with antibody as indicated in the left margin. Figures below band are protein quantities, normalized for actin content (not shown) and relative to CLTC RNAi control. The results shown are representative of three independent experiments.

\section{RESULTS}

\section{Lentiviral delivery of shRNAs targeted to a single Rho GTPase gene}

Several shRNAs - 2 shRNAs per targeted gene - were synthesized corresponding to the genes Rac1, Rac2 and Cdc42. Each shRNA also served as negative control for the two other, non-targeted genes. Furthermore, a shRNA targeting clathrin heavy chain (CLTC) was chosen as an additional control. To investigate the potency of the selected short hairpins to stably knock down their respective target, we designed lentiviral constructs expressing a shRNA from the pol III promoter H1 and an enhanced green fluorescent protein (EGFP) reporter from a $\mathrm{CMV}$ internal promoter. Both directions were possible for insertion of the siRNA cassette with equal capacity to knock down the target gene (data not shown). For simplicity, all the constructs were built with the internal promoters 5'-3' (Figure 1). Due to its consistent high Rac1, Rac2 and Cdc42 expression levels, the SupT1 T-cell line was chosen to verify the effectiveness of the shRNAs. Following transduction, cells were sorted for comparable EGFP levels, as shown for Rac2 RNAi in Figure 2. In a next step, Rac1, Rac2 and Cdc42 expression levels were assayed by Western blotting (Figure 3) and real-time PCR (Figure 4). For each gene the shRNA with the strongest knock-down was selected. As shown in Figure 3, in cells transduced with single shRNA expressing virus, targeted protein expression was 6 to 17 -fold less than in control transduced cells, while the other Rho GTPase proteins tested for were not affected, or showed an even higher expression level. A similar ablation was seen in mRNA expression (Figure 4). In conclusion, these assays showed a clear reduction of targeted gene expression, at mRNA and protein level, without diminishing that of the other Rho GTPases assayed for. This illustrates that in our experimental set-up the selected shRNAs were expressed at sufficient level to trigger stable, strong and gene-specific silencing.

\section{Simultaneous knock-down of 2 or 3 Rho GTPase genes}

To test the effectiveness of simultaneous knock-down of multiple genes, we inserted 2, and in a next step 3 RNAi cassettes $5^{\prime}-3^{\prime}$ into the TRIP vector (further referred to as tandem and triple RNAi). To this end, the H1-RNAi cassettes were consecutively EcoRI-MunI transferred from the pSUPER(MunI) vector into the EcoRI digested TRIP $\Delta$ U3-CMV-EGFP-WPRE vector. The restriction site EcoRI remains unique after ligation and can still be used to subclone additional H1-RNAi cassettes into the lentiviral transfer vector (Figure 1). Again, functionality of tandem and triple RNAi constructs was assayed by Western blotting and real-time PCR (Figure 3 and Figure 4) after sorting of transduced cells for comparable EGFP levels (Figure 2b). On the protein level, reduction in target gene expression was as strong as seen with single shRNA expressing viruses (Figure 3), although mRNA levels were less reduced (Figure 4). Similar experiments using the Jurkat T-cell line yielded the same results (data not shown). Overall, these experiments showed that multiple shRNAs, expressed from the same lentiviral vector, are present at sufficient level to knock down multiple targets. Knockdown persisted over at least 20 cell divisions, and lasted for some targets for over 1 month of continuous cell culture (data not shown).

\section{Functional implications of knock-down of Rho GTPases}

Rho GTPases are crucial proteins for directed cell migration (Raftopoulou and Hall, 2004). To investigate the functional correlate of the observed knock-down, we performed migration assays with SupT1 cells, known to respond chemotactically to stromal cell-derived factor-1 alpha (SDF-1 $\alpha$ (Secchiero et al. 1998). In these experiments, RNAi transduced cells were not sorted, allowing us to score migration in function of EGFP expression (gating on intensity regions as shown in Figure 2a, $E^{-}$to $E^{+++}$). As shown in Figure 5, knock-down of Rac1, Rac2 and Cdc42 all resulted in impaired chemotaxis towards SDF-1 $\alpha$. Inhibition of migration correlated with EGFP levels, indicating a biological read-out of the shRNA levels. Cells expressing shRNA migrated to a lower extent compared to cells not expressing shRNA when either one of the three Rho GTPases was knocked down, suggesting 
that targeted Rho GTPase proteins are all necessary for directional migration. Consequently, as expected from knock-down observed by immunoblot, this biological effect was retained in cells transduced with virus encoding multiple shRNAs (Figure 5).

\section{DISCUSSION}

In this report, we generated lentiviral constructs to study knock-down of Rho GTPases, important in cell motility. Simultaneous knock-down of several individual Rho GTPases was achieved with multiple siRNAs, each driven by individual $\mathrm{H} 1$ pol III promoters, expressed from a single lentiviral vector. Thanks to a separate marker gene insert, the dose-response on knock-down can be monitored.

In order to establish stable silencing of Rho GTPases, lentiviral constructs expressing shRNA were designed. Lentiviral gene transfer has been shown to be an efficient and gentle method to transfer siRNA into primary cells and by virtue of a marker gene, transduced cells effectively expressing shRNA can be identified. For effective knockdown of signaling cascades or redundant protein pathways, several targets have to be silenced in the same cell. In this study, we further extended the use of lentiviral siRNA transgenesis by generating tandem and triple constructs to achieve multiple protein knock-down. These constructs have an advantage over simultaneous transfection of several siRNAs, as the latter method does not ensure that all siRNAs are expressed simultaneously. Alternatively, multiple transductions with separate viruses, each encoding one shRNA, are feasible if high transduction efficiencies are attained. However, unless each vector encodes a different marker gene, it remains unclear if a single cell expresses all shRNAs intended to be transferred. Moreover, expression from a single vector has the advantage that the relative level of expression of each shRNA is constant. A method using plasmids expressing multiple siRNAs driven by U6 promoters to achieve knock down of escape mutants of a single target (Schubert et al. 2005) or to target different mRNAs (Jazag et al. 2005) were described recently. These and our results indicate that no apparent competition exists between multiple pol III promoters in close proximity and that no saturation of the RNA-induced silencing complex RISC was reached, as suggested previously (McManus et al. 2002). Moreover, Gonzalez et al. 2005 have recently reported a proportional improvement in HLA gene silencing by increasing the copy number of a selected U6shRNA cassette in the plasmid vector from 1 to 6 . Further increasing the number of RNAi cassettes showed a decrease in HLA down-regulation, evidencing a certain limit (competition or saturation) in shRNA expression capacity. However, this limit might be different depending on the targeted gene and the cell line or vector system that is used. Recently, reports have shown that simultaneous silencing of two different cellular targets is achievable using a single lentiviral vector containing two pol III promoters (Anderson and Akkina, 2005; Sumimoto et al. 2006). Our data corroborate these results and provide evidence that knock-down of three different targets from one type of pol III promoter is still feasible. Moreover, our design allows an easy gradual extension of the number of RNAi cassettes in the widely used lentiviral vector pTRIP, which can, based on its theoretical incorporation capacity (Verma and Somia, 1997), accommodate more than 10 shRNA cassettes. In this context of multiple identical promoter sequences in a row, the possibility of repeat sequencemediated deletion during reverse transcription should be considered. However, we hypothesize that the net effect of this phenomenon might be minimal when using high MOI. The evaluation of other and more sets of shRNAs is warranted and currently in progress.

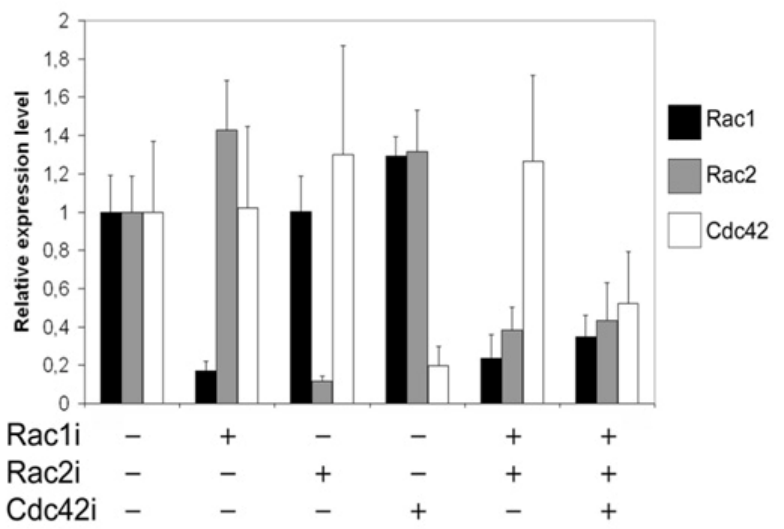

Figure 4. Messenger RNA expression of sorted SupT1 cells expressing specific RNAi as indicated. Bars indicate mean expression levels for Rac1 (black), Rac2 (grey) and Cdc42 (open); error bars indicate SD on at least three experiments. For comparison, levels are normalized to that of CLTC RNAi control.

Recently, nonspecific effects of siRNAs on gene expression have been reported (reviewed by Dykxhoorn and Lieberman, 2005). In our experiments, we did not observe 'off-target' stimulation or repression for the investigated genes. Some protein quantification results showed higher protein levels of non-targeted Rho GTPases. However, this effect was not observed on mRNA level. Moreover, Rac1 could not compensate for loss of function of Rac2 and vice versa in the chemotaxis assay. Interestingly, inhibition of migration correlated with EGFP levels, demonstrating that the marker gene expression, although driven by a separate promoter, is a reliable measure for shRNA levels. This validates our approach of sorting similar EGFP intensities in transduced cells for direct comparison of the effect of shRNA expression. Moreover, the potential caveat of comparing different vector stocks with different MOI is avoided by this approach. Further functional experiments are needed to delineate the Rho GTPase network response to knock-down of individual members using our vectors.

In conclusion, we present here a lentiviral shRNA delivery system that allows multiple shRNA species and a marker gene to be expressed from the same vector. The simultaneous knock-down of several targets within the same cell may be necessary, e.g. in compensatory or redundant pathways that have to be blocked at several levels, or to target hyper-variable viral sequences. Therefore, this strategy will likely prove to be of value in experimental and therapeutic applications. 


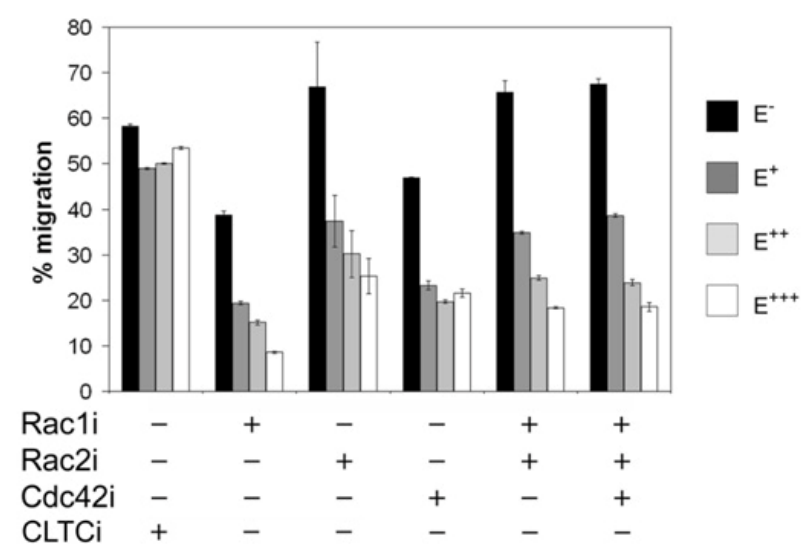

Figure 5. Chemotaxis of transduced SupT1 cells, expressing specific RNAi as indicated. CLTC RNAi is control. Bars indicate mean migration for four different expression intervals of EGFP (gated on $\mathrm{E}^{+}$to $\mathrm{E}^{+++}$, see Figure 2); error bars indicate $\mathrm{SD}$ on duplicate wells. The results shown are representative of three independent experiments.

\section{ACKNOWLEDGMENTS}

The authors thank Nancy De Cabooter and Els Demecheleer for technical assistance, Didier Trono (Université de Genève, Geneva, Switzerland) for the gift of the lentiviral packaging and envelope plasmids, Pierre Charneau (Hôpital Necker, Paris, France) for the gift of the lentiviral shuttle plasmid.

\section{REFERENCES}

ANDERSON, Joseph and AKKINA, Ramesh. HIV-1 resistance conferred by siRNA cosuppression of CXCR4 and CCR5 coreceptors by a bispecific lentiviral vector. AIDS Research and Therapy, January 2005, vol. 2, no. 1, p. 1.

ANDERSON, Joseph; BANERJEA, Akhil and AKKINA, Ramesh. Bispecific short hairpin siRNA constructs targeted to CD4, CXCR4, and CCR5 confer HIV-1 resistance. Oligonucleotides, October 2003, vol. 13, no. 5, p. 303-312.

BRUMMELKAMP, Thijn R.; BERNARDS, René and AGAMI, Reuven. A system for stable expression of short interfering RNAs in mammalian cells. Science, April 2002, vol. 296, no. 5567, p. 550-553.

CANTRELL, Doreen Ann. GTPases and T cell activation. Immunological Reviews, April 2003, vol. 192, no. 1, p. 122130.

DYKXHOORN, Derek M. and LIEBERMAN, Judy. The silent revolution: RNA interference as basic biology, research tool, and therapeutic. Annual Review of Medicine, February 2005, vol. 56, p. 401-423.

DYKXHOORN, D.M., NOVINA, C.D. and SHARP, P. A. Killing the messenger: short RNAs that silence gene expression. Nature Reviews Molecular Cell Biology, June
2003, vol. 4, no. 6, p. 457-467.

FITZGERALD, Anna; VAN KAN, Jan A.L. and PLUMMER, Kim M. Simultaneous silencing of multiple genes in the apple scab fungus, Venturia inaequalis, by expression of RNA with chimeric inverted repeats. Fungal Genetics and Biology, October 2004, vol. 41, no. 10, p. 963-971.

GONZALEZ, Sergio; CASTANOTTO, Daniela; LI, Haitang; OLIVARES, Simon; JENSEN, Michael C.; FORMAN, Stephen J.; ROSSI, John J. and COOPER, Laurence J.N. Amplification of RNAi-targeting HLA mRNAs. Molecular Therapy, May 2005, vol. 11, no. 5, p. 811-818.

JAZAG, Amarsanaa; KANAI, Fumihiko; IJICHI, Hideaki; TATEISHI, Keisuke; IKENOUE, Tsuneo; TANAKA, Yasuo; OHTA, Miki; IMAMURA, Jun; GULENG, Bayasi; ASAOKA, Yoshinari; KAWABE, Takao; MIYAGISHI, Makoto; TAIRA, Kazunari and OMATA, Massao. Single small-interfering RNA expression vector for silencing multiple transforming growth factor-beta pathway components. Nucleic Acids Research, August 2005, vol. 33, no. 15 , p. e131.

JI, Jingmin; WERNLI, Marion; KLIMKAIT, Thomas and ERB, Peter. Enhanced gene silencing by the application of multiple specific small interfering RNAs. FEBS Letters, September 2003, vol. 552, no. 2-3, p. 247-252.

KOVACIC, Hervé N.; IRANI, Kaikobad and GOLDSCHMIDT-CLERMONT, Pascal J. Redox regulation of human Rac1 stability by the proteasome in human aortic endothelial cells. Journal of Biological Chemistry, December 2001, vol. 276, no. 49, p. 4585645861.

LAKKA, Sajani S.; GONDI, Christopher S.; YANAMANDRA, Niranjan; OLIVERO, William C.; DINH, Dzung H.; GUJRATI, Meena and RAO, Jasti S. Inhibition of cathepsin B and MMP-9 gene expression in glioblastoma cell line via RNA interference reduces tumor cell invasion, tumor growth and angiogenesis. Oncogene, June 2004, vol. 23, no. 27, p. 4681-4689.

MCMANUS, Michael T.; HAINES, Brian B.; DILLON, Christopher P.; WHITEHURST, Charles E.; VAN PARIJS, Luk; CHEN, Jianzhu and SHARP, Phillip A. Small interfering RNA-mediated gene silencing in $T$ lymphocytes. The Journal of Immunology, November 2002, vol. 169 , no. 10, p. 5754-5760.

MOTLEY, Alison; BRIGHT, Nicholas A.; SEAMAN, Matthew N.J. and ROBINSON, Margaret S. Clathrinmediated endocytosis in AP-2-depleted cells. The Journal of Cell Biology, September 2003, vol. 162, no. 5, p. 909918.

NALDINI, Luigi; BLÖMER, Ulrike; GALLAY, Philippe; ORY, Daniel; MULLIGAN, Richard; GAGE, Fred H.; 
VERMA, Inder M. and TRONO, Didier. In vivo gene delivery and stable transduction of nondividing cells by a lentiviral vector. Science, April 1996, vol. 272, no. 5259, p. 263-267.

QIN, Xiao-Feng; AN, Dong Sung; CHEN, Irvin S.Y. and BALTIMORE, David. Inhibiting HIV-1 infection in human $T$ cells by lentiviral-mediated delivery of small interfering RNA against CCR5. Proceedings of the National Academy of Sciences of the United States of America, January 2003, vol. 100, no. 1, p. 183-188.

RAFTOPOULOU, Myrto and HALL, Alan. Cell migration: Rho GTPases lead the way. Developmental Biology, January 2004, vol. 265, no. 1, p. 23-32.

RUBINSON, Douglas A.; DILLON, Christopher P.; KWIATKOWSKI, Adam V.; SIEVERS, Claudia; YANG, Lili; KOPINJA, Johnny; ZHANG, Mingdi; MCMANUS, Michael T.; GERTLER, Frank B.; SCOTT, Martin L. and VAN PARIJS, Luk. A lentivirus-based system to functionally silence genes in primary mammalian cells, stem cells and transgenic mice by RNA interference. Nature Genetics, March 2003, vol. 33, no. 3, p. 401-406.

SCHUBERT, Steffen; GRUNERT, Hans-Peter; ZEICHHARDT, Heinz; WERK, Denise; ERDMANN, Volker A. and KURRECK, Jens. Maintaining inhibition: siRNA double expression vectors against coxsackieviral RNAs. Journal of Molecular Biology, February 2005, vol. 346, no. 2, p. 457-465.

SECCHIERO, P., ZELLA, D., BARABITSKAJA, O., REITZ, M.S., CAPITANI, S., GALLO, R.C. and ZAULI, G. Progressive and persistent downregulation of surface CXCR4 in CD4 ${ }^{+} \mathrm{T}$ cells infected with human herpesvirus 7. Blood, December 1998, vol. 92, no. 12, p. 4521-4528.

STOVE, Veronique; VAN DE WALLE, Inge; NAESSENS, Evelien; COENE, Elisabeth; STOVE, Christophe; PLUM, Jean and VERHASSELT, Bruno. Human immunodeficiency virus nef induces rapid internalization of the T-Cell coreceptor CD8 $\alpha \beta$. Journal of Virology, September 2005, vol. 79, no. 17, p. 11422-11433.

SUMIMOTO, Hidetoshi; HIRATA, Kenro; YAMAGATA, Shizuko; MIYOSHI, Hiroyuki; MIYAGISHI, Makoto; TAIRA, Kazunari and KAWAKAMI, Yutaka. Effective inhibition of cell growth and invasion of melanoma by combined suppression of BRAF (V599E) and Skp2 with lentiviral RNAi. International Journal of Cancer, January 2006, vol. 118, no. 2, p. 472-476.

VANDESOMPELE, J.; DE PRETER, Katleen; PATTYN, Filip; POPPE, Bruce; VAN ROY, Nadine; DE PAEPE, Anne and SPELEMAN, Frank. Accurate normalization of real-time quantitative RT-PCR data by geometric averaging of multiple internal control genes. Genome Biology, June 2002, vol. 3, no. 7, p. 0034 .

VERMA, Inder M. and SOMIA, Nikunj. Gene therapy- promises, problems and prospects. Nature, September 1997, vol. 389, no. 6648, p. 239-242.

YU, Jenn-Yah; TAYLOR, Jennifer; DERUITER, Stracy L.; VOJTEK, Anne B. and TURNER, David L. Simultaneous inhibition of GSK3 $\alpha$ and GSK3 $\beta$ using hairpin siRNA expression vectors. Molecular Therapy, February 2003, vol. 7, no. 2, p. 228-236.

ZUFFEREY, Romain; NAGY, Dea; MANDEL, Ron J.; NALDINI, Luigi and TRONO, Didier. Multiply attenuated lentiviral vector achieves efficient gene delivery in vivo. Nature Biotechnology, September 1997, vol. 15, no. 9, p. 871-875. 